\title{
Rare Ovarian Cancer Presentation
}

\section{Ricardo Pedrini Cruz ${ }^{1}$, Gustavo Peretti Rodini ${ }^{1}$, Margarete Duarte da Rosa ${ }^{1}$, Vinícius Duarte} Cabral$^{2}$, Eduardo Cambruzzi²

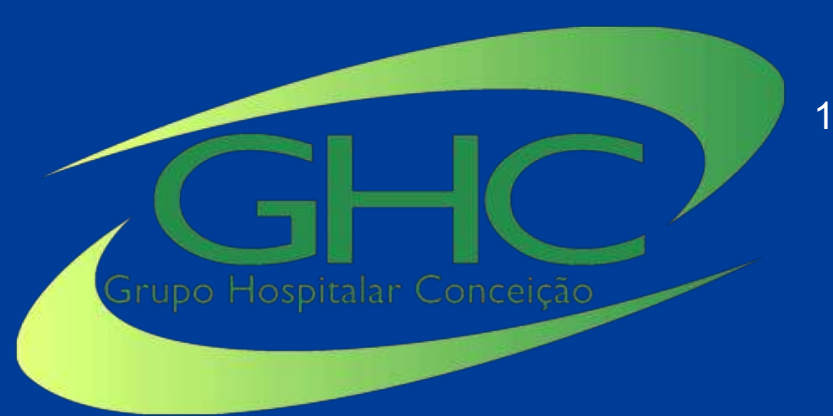

${ }^{1}$ Hospital Nossa Senhora da Conceição, Oncogynecology Dep., Porto Alegre, Brazil. ${ }^{2}$ Hospital Nossa Senhora da Conceição, Pathology Dep., Porto Alegre, Brazil.

\section{Objectives}

To report an intestinal lymphangitis carcinomatosa related to ovary cancer. Methods

The information has been obtained through review of medical records and review of the literature.

\section{Case Report}

A 57-year-old virgin, deaf-mute patient with cognitive deficit was referred to our hospital with left ovary neoplasia and palpable bilateral inguinal adenopathies. She had a CT abdomen performed about 7 months ago with a mixed lesion of the left ovary, with a diameter of $3.5 \mathrm{~cm}$. CA-125 was 74.55 , and the others tumours markers were normal.

Videolaparoscopy showed white ascites compatible with lymphatic fluid, lesions in epiploon, and absence of macroscopic changes in the small intestine. Left oophorectomy was performed. Frozen section revealed undifferentiated malignant neoplasia, not being able to confirm carcinoma or discard lymphoma. Late pathology sections confirmed the hypothesis of high grade serous ovarian carcinoma.

She was readmitted in the emergency 2 weeks later with high bowel obstruction. Abdominal CT showed stenosis of the proximal jejunum just after the fourth duodenal portion.

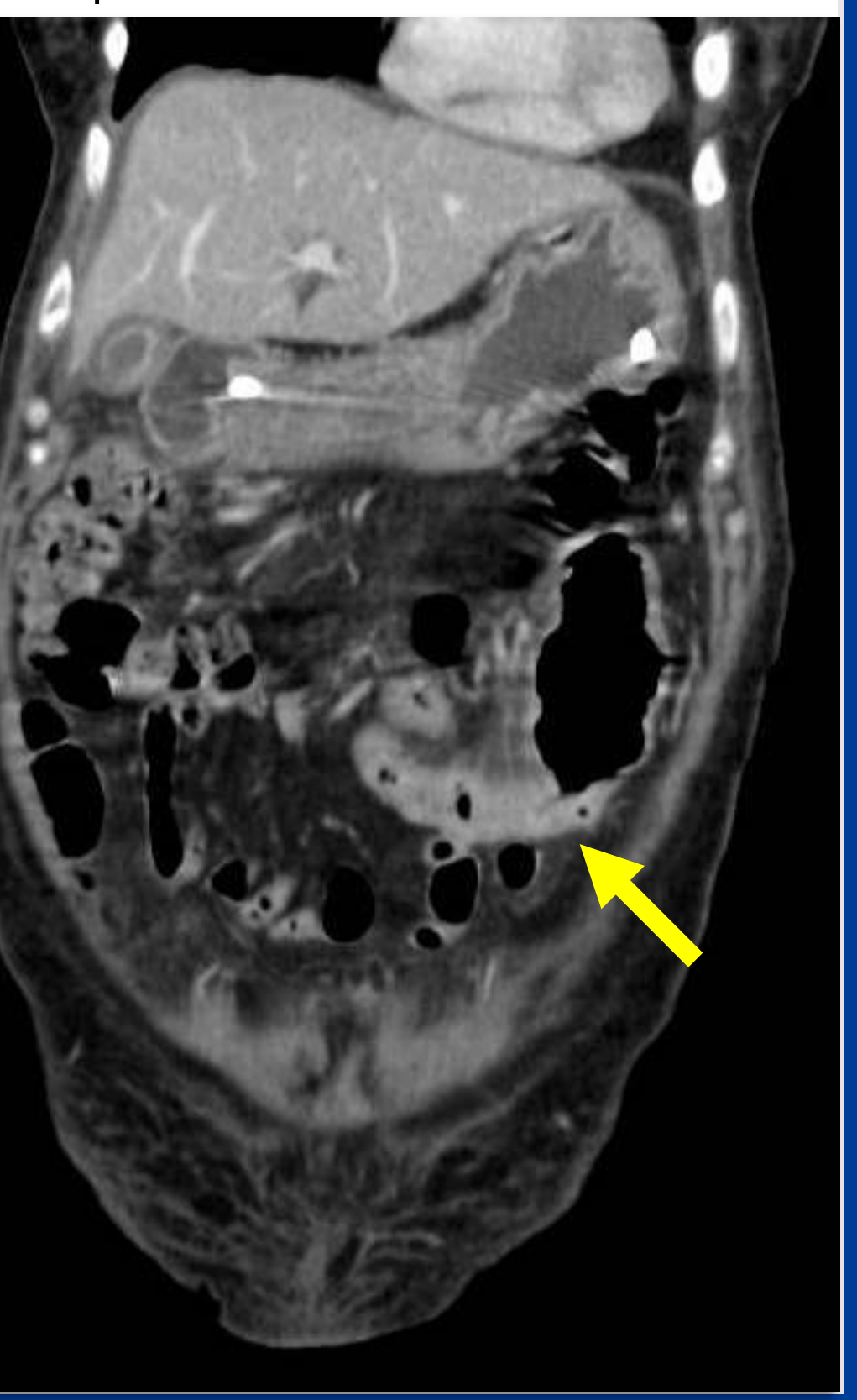

At laparotomy no ascites or lymphatic fluid was found. A thickening area of 10 $\mathrm{cm}$ in the proximal jejunum without intestinal peristalsis was observed, with stenotic enteric lumen, without implants in the serosa, with lesion apparently originating from its submucosal and muscular layers. Proximal enterectomy, panhysterectomy, omentectomy, and resection of peritoneal implants in hepatic round ligament and bladder were performed. Pathology examination in the jejunum: luminal stenosis due to an white, annular, infiltrating tumor, measuring $7.2 \mathrm{~cm}$ in length. Microscopic examination showed tumoral

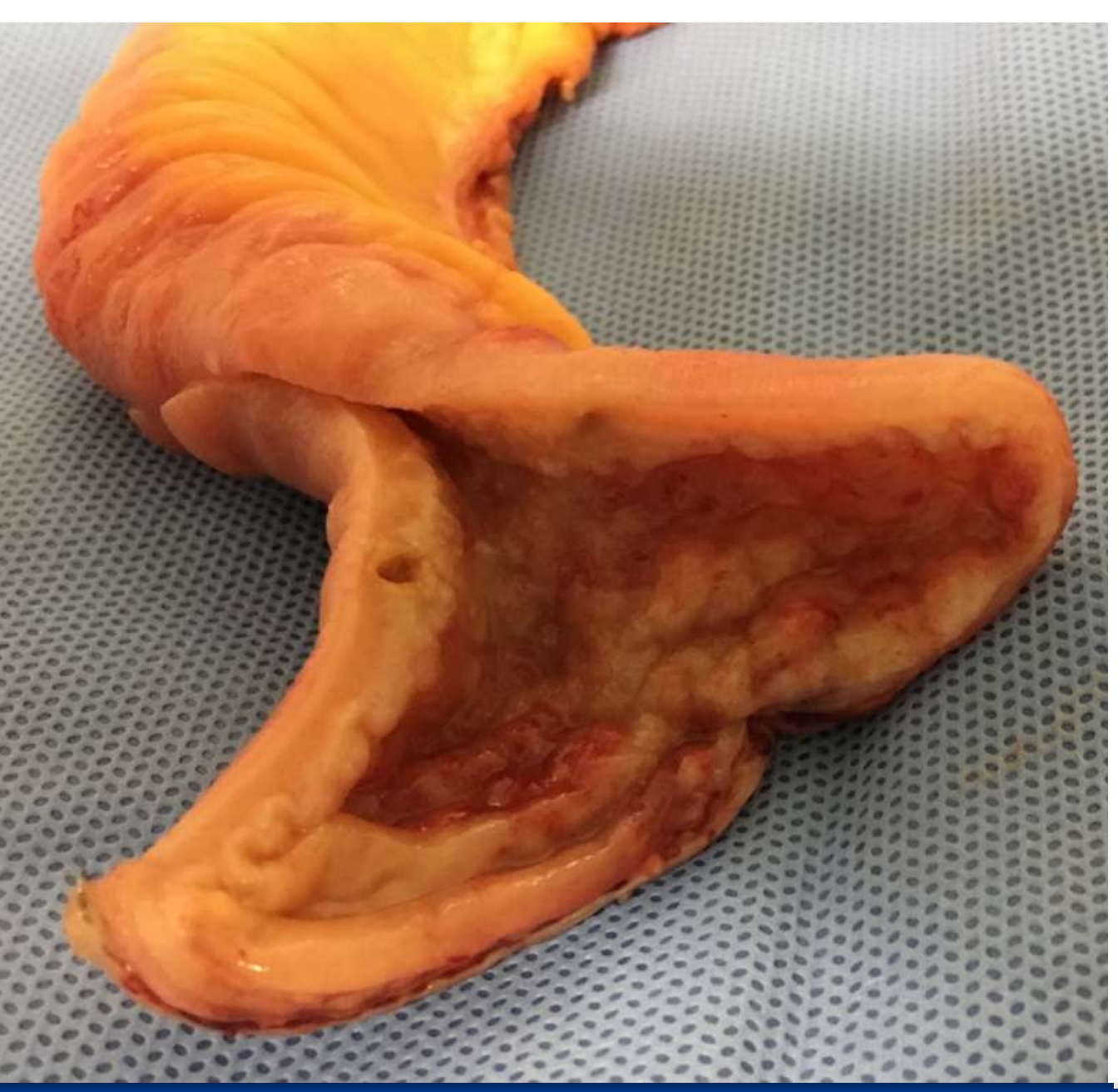
emboli in the lymphatics of the mucosa, submucosa and muscularis propria, associated with stromal invasion of these structures. No serosal involvement was identified in the jejunum.

Immunohistochemistry was positive for cytokeratin 7 (OV-TL 12/30), p63 (4A4), CA-125 (OC125), PAX-8 (MRQ-50), estrogen receptor (SP1), WT1 (6F-H2), and negative for cytokeratin 20 (Ks20.8), CDX2 EPR2764Y), Vilin (CWWB1) and GATA3 (L50-823); compatible with metastatic involvement of the small intestine by high grade serous ovarian carcinoma.

\section{Discussion}

Lymphangitis carcinomatosa is referred to as diffuse involvement of the metastatic malignant cells in lymph vessels (1). Extrapulmonary sites of lymphangitis

carcinomatosa originated in the ovary cancer have been rarely reported, such as the skin, duodenum, and bile duct (2-4). To the best of our knowledge, this is the first report in the literature of intestinal carcinomatous lymphangitis related to ovary cancer.

\section{References}

1- Bruce, D. M., S. D. Heys, and O. Eremin. Lymphangitis Carcinomatosa: A Literature Review. J R Coll Surg Edinb 1996;41(1):7-13.

2- Nakasono M, et al. Duodenal Lymphangitis Carcinomatosa: Endoscopic Characteristics and Clinical Significance. J Gastroenterol Hepatol 2006;21:79-83. 3- Keohane, S. G., et al. Cutaneous Lymphangitis Carcinomatosa: A Rare Primary Manifestation of Malignancy. J R Soc Med 1996;89(10):582-83.

4- Gabata, T., et al. Obstructive Jaundice Caused by Lymphangitis Carcinomatosa of Bile Duct Wall from Gastric Carcinoma. Abdom Imaging 1998;23(2):177-79.

\section{Contact}

Ricardo Pedrini Cruz - Department of Oncogynecology: Hospital Nossa Senhora da Conceição. Av. Francisco Trein, 596, Porto Alegre, RS, Brazil, 91350-200 Email: rpcruz2004@gmail.com 